\title{
Explicación de la Accidentabilidad Laboral Basada en Factores de Riesgo Psicosocial y Rasgos de Personalidad en el Transporte Forestal
}

\section{EXPLANATION OF OCCUPATIONAL ACCIDENTS BASED ON PSYCHOSOCIAL RISK FACTORS AND PERSONALITY TRAITS IN FORESTRY TRANSPORT}

\author{
Katherine Seguel Conejeros'1, Eduardo Navarrete Espinoza², Gabriela Bahamondes Valenzuela ${ }^{3}$ \\ 1. Ingeniero en Prevención de Riesgos, Licenciada en Ciencias de la Prevención de Riesgos. Los Ángeles, Chile. \\ 2. Académico e Investigador, Escuela de Ciencias y Tecnologías, Campus Los Ángeles, Universidad de Concepción, Los Ángeles, Chile. \\ 3. Psicólogo Organizacional, Campus Los Ángeles, Universidad de Concepción, Los Ángeles, Chile.
}

\section{RESUMEN}

Los conductores de transporte forestal trabajan por largos períodos de tiempo, lo que implica un incremento de fatiga y que, sumado a factores tanto personales como del medio, afectan la capacidad de respuesta ante reacciones inmediatas, pudiendo desencadenar eventos indeseados en la conducción. Por esto, se hace necesario indagar respecto a la personalidad de los individuos y su relación con el comportamiento que manifiestan en su entorno laboral. Se realizó un estudio en 2 empresas del rubro transporte forestal, con el objetivo de analizar la relación entre factores de riesgo psicosocial, el nivel de rasgos de personalidad que poseen los conductores y su posible efecto sobre la accidentabilidad. La muestra estuvo compuesta por 62 conductores, agrupados en dos clasificaciones, según condición de accidentabilidad, en accidentados y no accidentados. Se evaluó el riesgo psicosocial mediante el cuestionario SUSES0/ISTAS 21, y para los rasgos de personalidad, autoestima, impulsividad, locus de control y tensión se utilizaron test especializados; además, se realizó un registro sociodemográfico. Los resultados de la evaluación de riesgo psicosocial determinaron que ambas empresas estaban expuestas a riesgo bajo. Según condición de accidentabilidad, en ambos grupos se obtuvo igual nivel de autoestima y prevaleció la ausencia de estrés. Para la condición con accidente, la tendencia fue a la manifestación de niveles altos de impulsividad; y, para la condición sin accidente, a presentar locus de control externo. Se determinaron diferencias significativas, según condición de accidentabilidad, solo en la dimensión de riesgo psicosocial trabajo activo y desarrollo de habilidades. Para el grupo con accidentes, trabajo activo y desarrollo de habilidades fue la dimensión que presentó un mayor número de correlaciones significativas, tanto con otras dimensiones de riesgo psicosocial, así como también con estrés percibido; en cambio, en el grupo sin accidentes, el mayor número de correlaciones significativas se presentó entre doble presencia y los rasgos de personalidad impulsividad, locus de control y tensión.

(Seguel K, Navarrete E, Bahamondes G, 2017. Explicación de la Accidentabilidad Laboral Basada en Factores de Riesgo Psicosocial y Rasgos de Personalidad en el Transporte Forestal. Cienc Trab. SepDic; 19 [60]: 157-165).

Palabras Clave: ACCIDENTABILIDAD, RIESGO PSICOSOCIAL, RASGOS DE PERSONALIDAD, TRANSPORTE FORESTAL.

Correspondencia / Correspondence:

Katherine Seguel Conejeros

Universidad de Concepción

Escuela de Ciencias y Tecnologias, Campus Los Ángeles

Juan Antonio Coloma 0201, Los Ángeles, Chile

Tel. (56-43) 2405217

e-mail: kathseguel@gmail.com

Recibido: 05 de Septiembre de 2017 / Aceptado: 28 de Septiembre de 2017

\section{ABSTRACT}

The drivers of forestry transport work for long periods of time, which implies an increase in fatigue and which, added to both personal and environmental factors, affect the ability to respond to immediate reactions, which can trigger unwanted driving events. Therefore, it is necessary to inquire about the personality of individuals and their relationship with the behavior they manifest in their work environment. A study was carried out in 2 companies in the forest transport sector, with the aim of analyzing the relationship between psychosocial risk factors, the level of personality traits that drivers have and their possible effect on accident rates. The sample consisted of 62 drivers, grouped into two classifications, depending on accident conditions, in accidents and not accidents. Psychosocial risk was assessed using the SUSESO/ISTAS 21 questionnaire, and specialized tests were used for personality trais, self-esteem, impulsivity, locus of control and tension; In addition, a sociodemographic record was made. The results of the psychosocial risk assessment determined that both companies were exposed to low risk. Depending on accident condition, in both groups the same level of self-esteem was obtained and the absence of stress prevailed. For the accident condition, the tendency was to manifest high levels of impulsivity; and, for the condition without accident, to present locus of external control. Significant differences were determined, according to the accident rate, only in the psychosocial risk dimension, active work and skills development. For the group with accidents, active work and skills development, it was the dimension that presented a greater number of significant correlations, both with other dimensions of psychosocial risk, as well as with perceived stress; however, in the group without accidents, the greatest number of significant correlations occurred between double presence and personality traits impulsivity, locus of control and tension.

Key words: ACCIDENTABILITY, PSYCHOSOCIAL RISK, PERSONALITY TRAITS, FOREST TRANSPORT

\section{INTRODUCCIÓN}

Los accidentes son el resultado de actos erróneos que cometen los sujetos, o porque los equipos, herramientas, maquinarias o lugares de trabajo no se encuentran en condiciones adecuadas. El principio de la prevención de accidentes señala que todos los accidentes tienen causas que los originan y que se pueden evitar, identificando y controlando lo que produce dicho evento inde- 
seado; estas pueden ser: causas directas de origen humano (acción insegura, definida como cualquier acción o falta de acción de la persona que trabaja, lo que puede llevar a la ocurrencia de un accidente) y ambiental (condición insegura, definida como cualquier condición del ambiente laboral que puede contribuir a la ocurrencia de un accidente); y causas básicas de origen humano, que explican por qué la gente no actúa como debiera; y ambiental, que explican por qué existen condiciones inseguras ${ }^{1}$. En el mundo, una gran parte de las relaciones comerciales y económicas dependen de los medios de transporte terrestre para trasladar las materias primas, productos y subproductos, y los bienes necesarios para la mayoría de las actividades económicas que se desarrollan en un país ${ }^{2}$. Los conductores de transporte de carga que trabajan por largos períodos poseen un estilo de vida y conductas riesgosas, por lo que el chofer, bajo efecto de la fatiga, disminuye progresivamente la atención durante el manejo y pierde capacidad de respuesta ante condiciones específicas que exigen reacciones inmediatas cuando circula por las carreteras. Pestañear y dormitar durante el manejo son formas extremas de pérdida de la atención. Habitualmente, los accidentes producidos en estas circunstancias tienen alto costo en términos de personas, muertos, heridos y pérdidas ${ }^{3}$. Según la Superintendencia de Seguridad Social (SUSESO), al analizar las cifras por sector económico, la industria del Transporte presenta los mayores niveles de accidentabilidad, con una tasa de 6\% al año 2014; esto se debe a que la accidentabilidad en el transporte no sólo es el resultado de la gestión que el propio sector puede realizar, sino que es matizado por la influencia de la cultura de seguridad vial que el país presenta. Además, el ambiente en donde se desarrolla la actividad es difícil de controlar, ya que al utilizar las vías públicas de circulación, los trabajadores interactúan con peatones y otros conductores, quienes no necesariamente actúan de acuerdo a los estándares de seguridad 4 .

El aumento en el desarrollo de la tecnología para los puestos de trabajo, la amplitud de los mercados y la necesidad de trabajadores multifuncionales, han elevado el grado de carga mental al que están expuestos las personas, por sobre los riesgos físicos, en sectores como servicios, comercio y finanzas ${ }^{5}$. Estas condiciones del ambiente laboral afectan sobre los factores psicosociales, influyendo en el desempeño y comportamiento de los trabajadores. Según el Comité Mixto Organización Internacional del Trabajo -Organización Mundial de la Salud (OIT/OMS)-, los factores psicosociales consisten en interacciones entre el trabajo, el medio ambiente y las condiciones de organización; y, por otro lado, las capacidades del trabajador, sus necesidades, cultura y situación personal fuera del trabajo, todo lo cual, a través de percepciones y experiencias, pueden influir en la salud, el rendimiento y la satisfacción en el trabajo ${ }^{6}$. Estos factores, debido a una inadecuada calidad laboral, se expresan como riesgo psicosocial, el cual se define como todas las situaciones y condiciones del trabajo que se relacionan con el tipo de organización, el contenido del trabajo y la ejecución de la tarea, los cuales tienen la capacidad de afectar, en forma negativa, el bienestar y la salud (física, psíquica y/o social) del trabajador y sus condiciones de trabajo ${ }^{7}$. Según MINSAL ${ }^{7}$, los efectos o consecuencias que podrían causar los riesgos psicosociales en el trabajo serían los siguientes: efectos sobre la salud física de los trabajadores: cansancio, enfermedades cardiovasculares; trastornos músculo-esqueléticos; entre otros; efectos sobre la salud psicológica de los trabajadores: depresión, irritabilidad, insatisfacción, disminución de la capa- cidad del procesamiento de información y de respuesta, entre otras; y finalmente, efectos sobre los resultados del trabajo y sobre la propia organización: ausentismo laboral, masificación de licencias médicas, incremento de la siniestralidad o accidentes del trabajo, disminución en el rendimiento, productividad y calidad, entre otros.

En Chile, la Encuesta nacional de empleo, trabajo, salud y calidad de vida de los trabajadores y trabajadoras (ENETS 2009-2010) determinó que los principales síntomas que los chilenos asocian al trabajo son: sensación de cansancio, molestias oculares, dolores de cabeza, dificultad para dormir, tensiones o irritabilidad; siendo todos ellos síntomas de estrés ${ }^{8}$. El estrés, se entiende como la respuesta fisiológica, psicológica y del comportamiento del trabajador para intentar adaptarse a los estímulos que le rodean; pudiendo tener una repercusión en la salud del personal, pérdidas económicas y sociales que se presentarán en la empresa; dando origen a fenómenos novedosos como Síndrome de Burnout (quemarse por el trabajo), Mobbing (acoso laboral) y el Karoshi (muerte por exceso de trabajo) ${ }^{9}$.

El desarrollo de la humanidad ha sido impulsado por el trabajo; sin embargo, cada una de las funciones laborales está inserta en un ambiente propio, que genera ciertas condiciones, y que hacen que un trabajador se exponga en forma individual o colectiva a determinados factores de riesgo, los cuales favorecen la exposición a accidentes laborales, siendo los factores humanos la causa principal de su ocurrencia. Por lo anterior, se hace necesario ahondar en las características personales de los individuos y su relación con el comportamiento que manifiestan dentro de su entorno laboral. Los rasgos de personalidad corresponden a aquellas características psicológicas, emocionales y conductuales estables desde la edad adulta del individuo, que determina la manera de sentir, pensar, actuar y relacionarse, y las cuales, en su conjunto, constituyen la personalidad, que es una variable individual que caracteriza a la persona y que la diferencia de cualquier otra, determinando los modelos de responder en forma distinta ante las mismas situaciones ${ }^{10}$. La presencia de la idea o imagen sobrevalorada de las habilidades y la sensación de invulnerabilidad frente a un peligro por desconocimiento o incredulidad, pueden fomentar un control ineficiente de las propias emociones y conductas de los trabajadores, teniendo presente la facilidad que tienen algunas personas de pasar de un estado pasivo a la ira, al menor estímulo presente. Quizás, una baja autoestima puede repercutir en eventuales incidentes, debido a dos razones: los individuos pueden atribuir que los accidentes son merecidos; o porque evita una actitud proactiva frente a los riesgos existentes ${ }^{11}$. Otro factor es la baja capacidad de concentración y los niveles de tolerancia bajo presión en una tarea determinada, debido a una alta exigencia de sus líderes.

El presente estudio tuvo como objetivo general analizar la relación entre los posibles factores de riesgo psicosocial existentes, el nivel de rasgos de personalidad que poseen los trabajadores y su efecto sobre la accidentabilidad. Como objetivos específicos se propusieron:

i) determinar la existencia de factores de riesgo psicosocial en el entorno laboral;

ii) determinar el nivel de autoestima, impulsividad, locus de control y tensión que poseen los trabajadores en estudio; y finalmente,

iii) analizar la relación entre las dimensiones de riesgo psicosocial, el nivel de rasgos de personalidad que poseen los trabajadores y su efecto sobre la accidentabilidad. 


\section{MATERIALES Y MÉTODOS}

El presente estudio se enmarcó dentro de un diseño no experimental, de tipo transversal y correlacional. La población de estudio correspondió a conductores de transporte de carga, específicamente de los subsectores Forestal y Madera, cuyo cargamento corresponde a astillas, madera y trozas, y que cumplen su labor en la región del Biobío, Chile.

\section{Muestra}

La muestra constó de conductores voluntarios, los cuales fueron agrupados en dos clasificaciones: no accidentados y accidentados. Se consideraron dos tipos de muestras, no probabilística y probabilística; debido a que se realizó un muestreo intencional, que consistió en la elección de los individuos que se estimó fueron representativos para el estudio y que cumplieron con el criterio de inclusión, correspondiente a haber tenido un accidente. La selección del personal que no presentó accidentes se realizó en forma aleatoria. Para efectos de este estudio, se realizó un registro de variables socio-demográficas, tales como edad, género, estado civil, nivel de escolaridad, número de capacitaciones, tipo de contrato, tipo de turnos, antigüedad en el cargo, experiencia en el rubro y tipo de accidente, y su respectivo nivel de gravedad.

\section{Criterios de inclusión:}

- Personas que hubieran sufrido al menos un accidente laboral, ocurrido por acciones inseguras, exceptuando los accidentes de trayecto, en los dos últimos años.

- Personas no accidentadas que trabajaran en la empresa en estudio y que desempeñaran cargos similares con respecto a aquellos que hubieren tenido accidentes.

- Voluntarios que hubiesen firmado el consentimiento informado.

\section{Variables de estudio e instrumentos de medida}

Para llevar a cabo el estudio se utilizaron tests especializados, tanto para la evaluación de factores de riesgo psicosocial como para la evaluación de rasgos de personalidad.

\section{Factores de riesgo psicosocial}

La evaluación de factores de riesgo psicosocial se realizó a través del cuestionario SUSESO/ISTAS 21 versión breve, instrumento que evalúa, de carácter simple y a modo de diagnóstico, el grado de exposición a riesgo psicosocial al interior de una organización. Este cuestionario evalúa una agrupación de 5 dimensiones mayores: exigencias psicológicas en el trabajo: cuantitativas, cognitivas, emocionales, esconder emociones y sensoriales; trabajo activo y desarrollo de habilidades: posibilidades de desarrollo que tiene el trabajador, control sobre el tiempo de trabajo, sentido del trabajo, influencia e integración de la empresa; apoyo social en la empresa y calidad de liderazgo: claridad y conflicto de rol, calidad de liderazgo, relación con superiores y con compañeros de trabajo social; compensaciones: estima, inseguridad respecto al contrato y a las características en el trabajo; y finalmente, doble presencia: influencia de las preocupaciones domésticas sobre el trabajo y/o carga de trabajo doméstico ${ }^{7}$.

\section{Rasgos de personalidad}

Existen diversas maneras de evaluar la personalidad, dependiendo de la situación en que se encuentren; por ello, la personalidad está determinada por las vivencias, decisiones o crisis que el ser humano enfrenta y que debe resolver en cada etapa de desarrollo del ciclo vital; por lo cual, para el análisis de rasgos de personalidad, se determinó el nivel que poseían los trabajadores frente a cada rasgo, para lo cual se consideraron cuatro variables de estudio: autoestima, impulsividad, locus de control y tensión; evaluándose cada uno de ellos a través de los siguientes tests psicométricos:

- Autoestima: Escala de autoestima de Rosenberg, escala que consta de 10 afirmaciones de los sentimientos que tiene la persona sobre ella, 5 direccionadas positivamente y 5 negativamente; la graduación de respuestas tiene 4 puntos y se asigna el puntaje inverso a las afirmaciones direccionadas negativamente; los valores teóricos fluctúan entre 10 (baja autoestima) y 40 (alta autoestima), el puntaje de corte establecido fue según la mediana de la distribución de los datos, donde mayor o igual a 15 puntos se consideró con autoestima alta ${ }^{12}$.

- Impulsividad: Barrat Impulsiveness Scale (BIS - 11), consta de 30 preguntas de autoinforme, con cuatro opciones de respuesta relacionadas con la frecuencia de presentación del patrón conductual. Las puntaciones teóricas que un trabajador puede obtener en el BIS - 11 fluctúan entre 0 (puntaje mínimo) y 120 (puntaje máximo); el puntaje de corte establecido se basó en la mediana de la distribución de los datos, la que fue 30,5 , por lo que con 31 puntos se consideró impulsividad alta ${ }^{13}$.

- Locus de control: Escala de locus de control de Rotter, test de elección forzada que mide diferencias individuales en la atribución de las causas de sus conductas. En cada ítem se solicita a la persona que identifique la afirmación que se aproxima más a su forma de pensar. La escala consta de 29 ítems, con dos alternativas de respuesta, una que refleja el locus interno y otra el externo, el puntaje de corte establecido fue según la mediana de la distribución de los datos, donde mayor o igual a 8 puntos se considera que posee tendencia al locus de control externo ${ }^{14}$.

- Tensión: Escala de estrés percibido (PSS - 14), test diseñado para medir el grado en que los individuos evalúan situaciones de su vida como estresantes. La PSS - 14 incluye 14 preguntas con respuestas que varían entre 0 y 4 puntos para cada una. La baremación establecida para el test es: 1-16, ausencia de estrés; 17-21, estrés funcional; 22-26, estrés moderado; y 27-41, estrés agudo ${ }^{15}$.

\section{Análisis estadístico}

Se realizó un análisis descriptivo de ambas poblaciones bajo estudio (trabajadores accidentados y no accidentados), para obtener medidas de tendencia central y dispersión, además de las distribuciones de probabilidad de los datos. Se verificaron los supuestos de normalidad y homogeneidad de varianzas (pruebas de Shapiro Wilk's y F, respectivamente), determinándose que solo en la dimensión de riesgo psicosocial D2 (trabajo activo y desarrollo de habilidades), y en rasgos de personalidad, R2 (impulsividad) y R4 (tensión), se cumplió el supuesto de normalidad; por lo que se procedió a realizar transformaciones Box-Cox para las variables restantes, en las que se logró cumplir con la normalidad de los datos con las siguientes transformaciones: log D1 (exigencias psicológicas), log D4 (compensaciones) para riesgo psicosocial, y para el rasgo de personalidad log R1 (autoestima). Se realizó la comparación de las diferentes puntuaciones asociadas a las dimensiones de riesgo psicosocial y de rasgos de personalidad, en ambos grupos, a través de una prueba t para muestras independientes ${ }^{16}$. Para las dimensiones D3 (apoyo social y calidad de liderazgo) y D5 (doble presencia), y para el rasgo de personalidad 
R3 (locus de control), no se logró cumplir con el supuesto de normalidad; en dichos casos, se utilizó la prueba no paramétrica para datos independientes de U Mann-Whitney ${ }^{17}$. La relación entre las diferentes variables cuantitativas, tanto para el registro socio-demográfico, como en la determinación de riesgo psicosocial y de los rasgos de personalidad, se evaluó a través de una matriz de coeficientes de correlación de Kendall (caso no paramétrico $)^{18}$.

\section{RESULTADOS}

La muestra estuvo compuesta por un total de 62 conductores voluntarios del género masculino, pertenecientes a 2 empresas; 30 conductores de la empresa A, con dos áreas de trabajo (madera y forestal (astilla)), y 32 conductores de la empresa B, con solo un área de trabajo (forestal (trozas)). Para los efectos del estudio se definieron 2 grupos: sin accidentes y con accidentes (31 casos por grupo) (Tabla 1).

La totalidad de los conductores desarrollaban sus actividades, tanto en régimen diurno como nocturno, y poseían contrato de tipo indefinido. La edad de los trabajadores evaluados fluctuó entre 28 y 67 años (promedio 44,5 años), una antigüedad en el cargo de 5,9 y 16,5 años de experiencia en el rubro como promedio, respectivamente. Cabe destacar la similitud entre los grupos bajo estudio, en cada una de las variables mencionadas (Tabla 2).

Tabla 1.

Caracteristicas de la muestra, según condición sin accidente/con accidente.

\begin{tabular}{lrrrrrr} 
& \multicolumn{2}{c}{ Sin Accidente } & \multicolumn{2}{c}{ Con Accidente } & \multicolumn{2}{c}{ Total } \\
& N & $\%$ & N & \multicolumn{1}{c}{$\%$} & \multicolumn{1}{c}{ N } & \multicolumn{1}{c}{$\%$} \\
Casos & 31,0 & 50,0 & 31,0 & 50,0 & 62,0 & 100,0 \\
Distribución según carga de trabajo & & & & & \\
Madera & 3,0 & 9,7 & 3,0 & 9,7 & 6,0 & 9,7 \\
Forestal (astilla) & 12,0 & 38,7 & 12,0 & 38,7 & 24,0 & 38,7 \\
Forestal (trozas) & 16,0 & 51,6 & 16,0 & 51,6 & 32,0 & 51,6 \\
Distribución según rango de edad & & & & & \\
20-40 & 11,0 & 35,5 & 8,0 & 25,8 & 19,0 & 30,6 \\
41-60 & 19,0 & 61,3 & 22,0 & 71,0 & 41,0 & 66,1 \\
>60 & 1,0 & 3,2 & 1,0 & 3,2 & 2,0 & 3,3 \\
Distribución según nivel de estudios & & & & & \\
Básica completa & 6,0 & 19,4 & 1,0 & 3,2 & 7,0 & 11,3 \\
Básica incompleta & 0,0 & 0,0 & 2,0 & 6,5 & 2,0 & 3,2 \\
Media completa & 14,0 & 45,2 & 12,0 & 38,7 & 26,0 & 41,9 \\
Media incompleta & 9,0 & 29,0 & 12,0 & 38,7 & 21,0 & 33,9 \\
Superior completa & 1,0 & 3,2 & 1,0 & 3,2 & 2,0 & 3,2 \\
Superior incompleta & 1,0 & 3,2 & 3,0 & 9,7 & 4,0 & 6,5 \\
Distribución según estado civil & & & & & & \\
Soltero (a) & 7,0 & 22,6 & 4,0 & 12,9 & 11,0 & 17,7 \\
Casado (a) & 23,0 & 74,2 & 24,0 & 77,4 & 47,0 & 75,8 \\
Separado (a) & 0,0 & 0,0 & 1,0 & 3,2 & 1,0 & 1,6 \\
Divorciado (a) & 1,0 & 3,2 & 2,0 & 6,5 & 3,0 & 4,8
\end{tabular}

Tabla 2.

Caracterización de la edad, tiempo en el cargo y antigüedad en el rubro, según condición sin accidente/con accidente.

\begin{tabular}{lcccccc} 
& \multicolumn{2}{c}{ Sin Accidente } & \multicolumn{3}{c}{ Con Accidente } & \multicolumn{2}{c}{ Total } \\
& Promedio & D.E. & Promedio & D.E. & Promedio & D.E. \\
Edad (años) & 43,5 & 7,9 & 45,4 & 8,1 & 44,5 & 8,0 \\
Cargo (años) & 5,7 & 3,4 & 6,0 & 3,7 & 5,9 & 3,5 \\
Rubro (años) & 17,1 & 7,7 & 15,8 & 7,1 & 16,5 & 7,4
\end{tabular}

Con D.E.: Desviación estándar.

\section{Riesgo psicosocial en las empresas en estudio}

En la empresa A, las dimensiones doble presencia (5) y compensaciones (4), concentran los mayores porcentajes asociados a un nivel de riesgo alto (color rojo) (Figura 1); y para la empresa B, las dimensiones doble presencia (5), exigencias psicológicas (1) y compensaciones (4) (Figura 2). En la empresa A, la dimensión 2 (trabajo activo y desarrollo de habilidades) se encuentra con un 60\% de riesgo bajo, constituyendo la situación más favorable para la salud de los conductores. El riesgo medio predominó en la dimensión 3 (apoyo social en la empresa y calidad del liderazgo) con 50\% de nivel de exposición. En las dimensiones 4 (compensaciones) y 5 (doble presencia), el nivel de riesgo medio se encuentra latente, con un 47\% y 40\%, respectivamente (Figura 1). Asimismo, en la empresa B, y al igual que en la empresa A, la condición más favorable para la salud de los trabajadores es la dimensión 2, con un 69\% de nivel de exposición a riesgo bajo. Las dimensiones 3 y 4 poseen un 42\% de riesgo medio; en cambio, en la dimensión 5, el nivel de exposición es de un $42 \%$ de riesgo alto; sin embargo, este porcentaje no permite calificar a la muestra de la empresa con exposición a riesgo psicosocial alto (Figura 2).

Los resultados de la evaluación determinan que, según las condiciones de ambas organizaciones, y de acuerdo a lo establecido en el

Figura 1.

Semáforo de riesgo psicosocial de la empresa $A$.

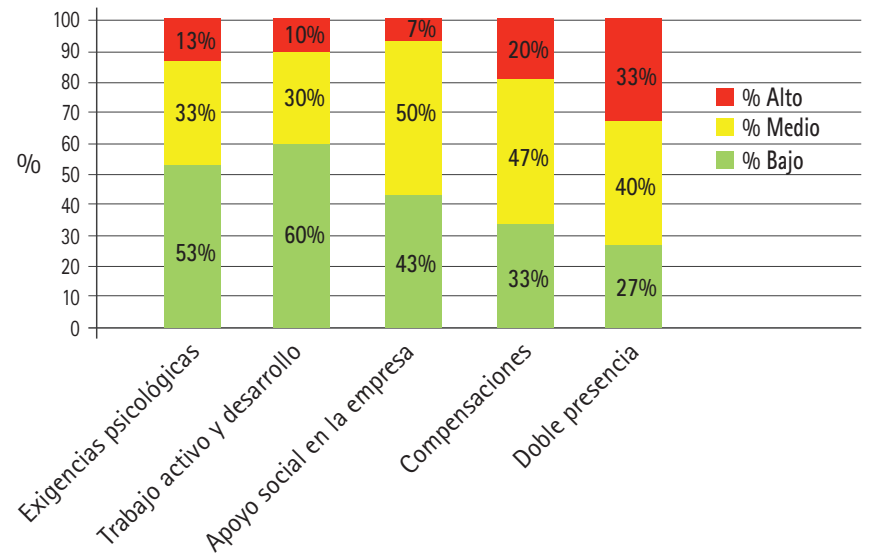

Figura 2.

Semáforo de riesgo psicosocial de la empresa B.

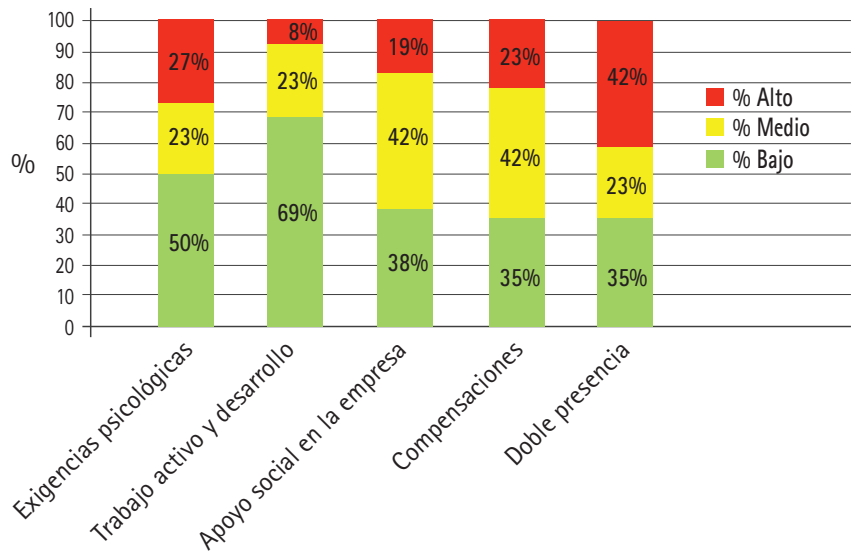


Tabla 3.

Nivel de exposición a riesgo psicosocial de cada empresa, según condición sin accidente o con accidente.

\begin{tabular}{|c|c|c|c|c|c|c|c|c|c|c|c|c|}
\hline & \multicolumn{6}{|c|}{ Empresa A } & \multicolumn{6}{|c|}{ Empresa B } \\
\hline & B (\%) & M (\%) & $A(\%)$ & B (\%) & $\mathrm{M}(\%)$ & $A(\%)$ & B (\%) & $\mathrm{M}(\%)$ & $A(\%)$ & B (\%) & $\mathrm{M}(\%)$ & $A(\%)$ \\
\hline Exigencias psicológicas & 60,0 & 27,0 & 13,0 & 47,0 & 40,0 & 13,0 & 56,0 & 19,0 & 25,0 & 38,0 & 31,0 & 31,0 \\
\hline Apoyo social en la empresa y calidad del liderazgo & 46,7 & 40,0 & 13,0 & 40,0 & 60,0 & 0,0 & 31,0 & 44,0 & 25,0 & 44,0 & 38,0 & 19,0 \\
\hline Compensaciones & 40,0 & 40,0 & 20,0 & 27,0 & 53,0 & 20,0 & 25,0 & 56,0 & 19,0 & 38,0 & 31,0 & 31,0 \\
\hline Doble presencia & 26,7 & 53,0 & 20,0 & 27,0 & 27,0 & 47,0 & 13,0 & 44,0 & 44,0 & 44,0 & 19,0 & 38,0 \\
\hline
\end{tabular}

Con B: nivel bajo; M: nivel medio y A: nivel alto.

protocolo de vigilancia de riesgos psicosociales, dado que de las 5 dimensiones evaluadas solo una se encuentra superior al 50\% de riesgo bajo, permite calificar a las muestras de ambas empresas con riesgo bajo; por otro lado, de la totalidad de dimensiones consideradas en la evaluación, ninguna se encuentra en riesgo mayor al $50 \%$, lo que no permite calificar a las empresas con riesgo alto. Según lo anterior, las poblaciones bajo estudio no presentan riesgo psicosocial; además, los niveles de riesgo bajo en la dimensión trabajo activo y desarrollo de habilidades, permiten desarrollar factores protectores, situación que permite calificar a la población como expuestos a riesgo psicosocial nivel bajo.

\section{Riesgo psicosocial por condición de accidentabilidad}

Los resultados obtenidos para la empresa A, muestran que la dimensión más crítica (> 50\%) para el grupo sin accidente, es la dimensión 5 (doble presencia), que califica al grupo con riesgo medio, debido a que posee un 53\% de nivel de exposición; las situaciones más críticas en el grupo con accidente, son las dimensiones 3 (apoyo social) y 4 (compensaciones), con un 60\% y 53\% de riesgo medio respectivamente; en cambio, en la Empresa B, existe sólo una dimensión con riesgo psicosocial medio, la dimensión 4 (compensaciones), que posee un 56\% de exposición, perteneciente al grupo sin accidente (Tabla 3).

\section{Rasgos de personalidad por grupos}

Ambos grupos bajo estudio presentaron porcentajes iguales en autoestima; el grupo con accidente posee una mayor frecuencia en el nivel de impulsividad alto; ambas condiciones de accidentabilidad tienen tendencia al locus de control externo; y en el caso de tensión, el grupo sin accidente, posee un porcentaje mayor de conductores con un nivel de estrés agudo, a diferencia del grupo con accidente, en el que predomina el estrés funcional (Tabla 4).

\section{Distribución de puntajes obtenidos para riesgo psicoso- cial y rasgos de personalidad}

Según la distribución de los puntajes obtenidos para cada dimensión de riesgo psicosocial evaluada, se presentaron diferencias significativas entre grupos solo en la dimensión 2 (trabajo activo

Tabla 4.

Nivel de rasgo de personalidad, según condición de accidentabilidad.

\begin{tabular}{lcccc} 
& \multicolumn{2}{c}{ Sin Accidente } & \multicolumn{2}{c}{ Con Accidente } \\
& B (\%) & A (\%) & B (\%) & A (\%) \\
Autoestima & 45,2 & 54,8 & 45,2 & 54,8 \\
Impulsividad & 51,6 & 48,4 & 48,4 & 51,6 \\
Locus de Control & 38,7 & 61,3 & 41,9 & 58,1
\end{tabular}
AE (\%) EF (\%) EM (\%) EA (\%) AE (\%) EF (\%) EM (\%) EA (\%)

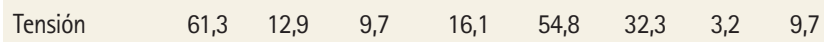
Con B: bajo; A: Alto; AE: ausencia de estrés; EF: estrés funcional; EM: estrés moderado y EA: estrés agudo.
Tabla 5.

Puntajes promedios para cada dimensión del Cuestionario SUSESOISTAS 21 por condición de grupo.

\begin{tabular}{|c|c|c|c|c|c|c|}
\hline & \multicolumn{3}{|c|}{ Sin Accidente } & \multicolumn{3}{|c|}{ Con Accidente } \\
\hline & $\begin{array}{l}\text { Puntaje } \\
\text { promedio }\end{array}$ & & $\begin{array}{l}\text { Nivel } \\
\text { de riesgo }\end{array}$ & $\begin{array}{l}\text { Puntaje } \\
\text { promedio }\end{array}$ & D.E. & $\begin{array}{l}\text { Nivel } \\
\text { de riesgo }\end{array}$ \\
\hline $\begin{array}{l}\text { Exigencias } \\
\text { psicológicas }\left(D_{1}\right)\end{array}$ & $8,90^{a}$ & 2,44 & M & $9,52^{\mathrm{a}}$ & 2,78 & M \\
\hline $\begin{array}{l}\text { Trabajo activo y desarrollo } \\
\text { de habilidades }\left(D_{2}\right)\end{array}$ & $3,81^{a}$ & 2,34 & B & $5,19^{b}$ & 2,34 & M \\
\hline $\begin{array}{l}\text { Apoyo social en la empresa } \\
\text { y calidad del liderazgo }\left(D_{3}\right)\end{array}$ & $\left(^{*}\right) 4,26^{a}$ & 2,38 & M & $4,10^{\mathrm{a}}$ & 2,47 & B \\
\hline Compensaciones (D4) & $4,00^{\mathrm{a}}$ & 2,93 & $M$ & $4,16^{a}$ & 2,91 & M \\
\hline Doble presencia $\left(D_{5}\right)$ & $\left.{ }^{*}\right) 2,68^{\mathrm{a}}$ & 1,62 & M & $2,61^{a}$ & 1,80 & M \\
\hline
\end{tabular}

En filas, letras iguales no difieren estadisticamente $(p>0,05)$. Con nivel de riesgo B: bajo y M: medio. D.E.: desviación estándar. ( $\left.{ }^{*}\right)$ Prueba no paramétrica de U-Mann Whitney.

y desarrollo de habilidades), presentando el grupo con accidentes, un puntaje estadísticamente superior en esa dimensión con respecto al grupo sin accidentes (diferencia de 1,38 puntos), clasificándose al primero con un nivel de riesgo medio. Para el resto de las dimensiones no se presentaron diferencias significativas entre ambos grupos en estudio (Tabla 5).

Las diferentes variables que caracterizan los rasgos de personalidad no presentaron diferencias significativas entre ambos grupos bajo estudio; sin embargo, el grupo con accidente manifestó una tendencia a mayor impulsividad y menor autoestima; y el grupo sin accidente, hacia locus de control externo (Tabla 6).

Tabla 6.

Puntajes promedios de test para cada rasgo de personalidad.

\begin{tabular}{lccccc} 
& \multicolumn{3}{c}{ Sin Accidente } & \multicolumn{2}{c}{ Con Accidente } \\
& Puntaje promedio & D.E. & Puntaje promedio & D.E. \\
Autoestima $\left(R_{1}\right)$ & $15,61^{\mathrm{a}}$ & 3,55 & $15,35^{\mathrm{a}}$ & 3,54 \\
Impulsividad $\left(\mathrm{R}_{2}\right)$ & $30,84^{\mathrm{a}}$ & 8,02 & $31,68^{\mathrm{a}}$ & 8,99 \\
Locus de control $\left(\mathrm{R}_{3}\right)$ & $\left(^{*}\right) 8,06^{\mathrm{a}}$ & 2,24 & $7,94^{\mathrm{a}}$ & 2,53 \\
Tensión $\left(\mathrm{R}_{4}\right)$ & $15,77^{\mathrm{a}}$ & 8,11 & $14,74^{\mathrm{a}}$ & 7,55
\end{tabular}

En filas, letras iguales no difieren estadisticamente $(p>0,05)$. Con D.E.: desviación estándar. (*) Prueba no paramétrica de U-Mann Whitney.

\section{Relación entre variables socio-demográficas, dimensiones de riesgo psicosocial y rasgos de personalidad}

Se presentaron correlaciones significativas en el grupo con accidente, entre la edad y rubro (a mayor edad, mayor experiencia en el rubro); edad y $\mathrm{R}_{1}$ (a mayor edad, tendencia a la autoestima alta); cargo y rubro (a mayor antigüedad en el cargo, mayor experiencia en el rubro); $\mathrm{D}_{3} \mathrm{y}, \mathrm{D}_{1}, \mathrm{D}_{2}, \mathrm{D}_{4} \mathrm{y} \mathrm{R}_{4}$, esto indica que a mayor apoyo social, mayor son las exigencias psicológicas que poseen los conductores, mayor será la oportunidad de desarrollar sus habilidades, poseen mejores compensaciones y aumentará el nivel de tensión (estrés percibido) que posean los conductores; rubro y $\mathrm{R}_{1}$, a mayor expe- 
Tabla 7.

Correlaciones entre variables socio-demográficas, dimensiones de riesgo psicosocial y rasgos de personalidad, condición con accidente.

\begin{tabular}{|c|c|c|c|c|c|c|c|c|c|c|c|c|}
\hline & Edad & Cargo & Rubro & $\mathrm{D}_{1}$ & $D_{2}$ & $D_{3}$ & $\mathrm{D}_{4}$ & $D_{5}$ & $R_{1}$ & $\mathrm{R}_{2}$ & $\mathrm{R}_{3}$ & $\mathrm{R}_{4}$ \\
\hline Edad & 1,00 & & & & & & & & & & & \\
\hline Rubro & 0,41 & 0,28 & 1,00 & & & & & & & & & \\
\hline $\mathrm{D}_{2}$ & $-0,10$ & $-0,05$ & $-0,03$ & 0,06 & 1,00 & & & & & & & \\
\hline $\mathrm{D}_{3}$ & $-0,04$ & $-0,13$ & $-0,03$ & 0,31 & 0,29 & 1,00 & & & & & & \\
\hline $\mathrm{D}_{4}$ & $-0,22$ & $-0,10$ & $-0,16$ & 0,15 & 0,25 & 0,57 & 1,00 & & & & & \\
\hline $\mathrm{R}_{1}$ & 0,31 & $-0,02$ & 0,26 & $-0,11$ & $-0,02$ & 0,18 & 0,06 & 0,11 & 1,00 & & & \\
\hline $\mathrm{R}_{2}$ & 0,04 & 0,12 & $-0,05$ & 0,17 & 0,01 & 0,15 & 0,07 & 0,12 & 0,05 & 1,00 & & \\
\hline $\mathrm{R}_{3}$ & $-0,06$ & 0,06 & 0,12 & 0,14 & 0,04 & 0,21 & 0,29 & 0,00 & 0,20 & $-0,09$ & 1,00 & \\
\hline $\mathrm{R}_{4}$ & $-0,06$ & $-0,18$ & 0,01 & 0,25 & 0,25 & 0,34 & 0,15 & 0,17 & 0,07 & 0,31 & $-0,02$ & 1,00 \\
\hline
\end{tabular}

Valores en rojo indican correlaciones significativas (Test de Kendall, $p<0,05$ ).

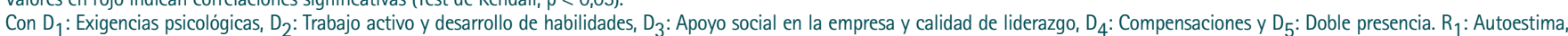
$\mathrm{R}_{2}$ : Impulsividad, $\mathrm{R}_{3}$ : Locus de control y $\mathrm{R}_{4}$ : Tensión.

riencia en el rubro, tendencia a la autoestima elevada; $\mathrm{D}_{2} \mathrm{y}, \mathrm{D}_{4} \mathrm{y} \mathrm{R}_{4}$, a mayor trabajo activo y desarrollo de habilidades, las compensaciones y nivel de tensión aumentarán; $\mathrm{R}_{3} \mathrm{y} \mathrm{D}_{4}$, cuando existe un mayor apoyo por parte de los líderes, mayores son las compensaciones que reciben los conductores; $\mathrm{D}_{4} \mathrm{y} \mathrm{R}_{3}$, al aumentar las compensaciones, existe una tendencia al locus de control externo; $\mathrm{R}_{2} \mathrm{y} \mathrm{R}_{4}$, a mayor nivel de impulsividad mayor estrés percibido por los conductores (Tabla 7).

Para el grupo sin accidentes se presentaron correlaciones significativas entre edad y cargo, rubro y $\mathrm{R}_{1}$; esto implica que a medida que aumenta la edad, aumenta la antigüedad en el cargo, la experiencia en el rubro y el nivel de autoestima; $\mathrm{D}_{1} \mathrm{y} \mathrm{D}_{3}$, a mayor exigencia psicológica en el trabajo mayor es el apoyo social en la empresa y la existencia de liderazgo por la jefatura; $D_{5} \mathrm{y} \mathrm{R}_{2}$, a mayor preocupación por la familia y las labores domésticas, mayor es la impulsividad; $\mathrm{R}_{3} \mathrm{y} \mathrm{D}_{5}$, a mayor tendencia al locus de control externo, mayor es la preocupación por la familia y las labores domésticas; $\mathrm{y} \mathrm{R}_{4} \mathrm{y}_{5}$, entre mayor es el nivel de estrés percibido, mayor es la preocupación por la familia y las labores domésticas; $\mathrm{R}_{1} \mathrm{y} \mathrm{R}_{4}$, a mayor nivel de autoestima, mayor será el nivel de tensión que exista; y $\mathrm{R}_{2} \mathrm{y}_{3}$, a mayor impulsividad, existe una tendencia al locus de control externo (Tabla 8).
La relación que existe entre: cargo y $\mathrm{D}_{3}$, rubro y $\mathrm{D}_{1}$ y rubro y $\mathrm{D}_{5}$, es inversamente proporcional, lo que indica que a mayor antigüedad en el cargo disminuye el apoyo social y calidad de liderazgo en las organizaciones, a mayor experiencia en el rubro, menor son las exigencias laborales; y a mayor experiencia en el rubro menor son las preocupaciones familiares y domésticas, esto se puede deber a la costumbre que poseen los conductores que tienen más experiencia a estar lejos de sus familias, o viceversa (Tabla 8).

Cabe destacar que para el caso del grupo con accidentes, la variable $\mathrm{D}_{2}$ (trabajo activo y desarrollo de habilidades) fue la dimensión que presentó un mayor número de correlaciones significativas, tanto con otras dimensiones de riesgo psicosocial, así como también con el estrés percibido ( $\mathrm{R}_{4}$, tensión). En cambio, el grupo sin accidentes, el mayor número de correlaciones significativas se presentó entre $\mathrm{D}_{5}$ (doble presencia) y los rasgos de personalidad impulsividad $\left(\mathrm{R}_{2}\right)$, locus de control $\left(\mathrm{R}_{3}\right)$ y tensión $\left(\mathrm{R}_{4}\right)$.

\section{DISCUSIÓN}

\section{Riesgo psicosocial según condición de accidentabilidad}

Los resultados obtenidos en esta investigación refutan lo determi-

Tabla 8.

Correlaciones entre variables sociodemográficas, dimensiones de riesgo psicosocial y rasgos de personalidad, condición sin accidente.

\begin{tabular}{|c|c|c|c|c|c|c|c|c|c|c|c|c|}
\hline & Edad & Cargo & Rubro & $\mathrm{D}_{1}$ & $D_{2}$ & $D_{3}$ & $\mathrm{D}_{4}$ & $D_{5}$ & $\mathrm{R}_{1}$ & $\mathrm{R}_{2}$ & $\mathrm{R}_{3}$ & $\mathrm{R}_{4}$ \\
\hline Rubro & 0,51 & 0,20 & 1,00 & & & & & & & & & \\
\hline $\mathrm{D}_{2}$ & $-0,17$ & 0,00 & $-0,16$ & 0,21 & 1,00 & & & & & & & \\
\hline $\mathrm{D}_{3}$ & $-0,17$ & $-0,27$ & $-0,15$ & 0,42 & $-0,11$ & 1,00 & & & & & & \\
\hline $\mathrm{D}_{4}$ & $-0,13$ & $-0,08$ & $-0,04$ & 0,03 & 0,07 & 0,01 & 1,00 & & & & & \\
\hline $\mathrm{R}_{2}$ & 0,04 & $-0,03$ & 0,01 & 0,20 & 0,07 & 0,01 & 0,01 & 0,25 & 0,00 & 1,00 & & \\
\hline $\mathrm{R}_{3}$ & $-0,03$ & $-0,10$ & 0,01 & 0,05 & 0,03 & 0,05 & $-0,02$ & 0,42 & 0,16 & 0,27 & 1,00 & \\
\hline $\mathrm{R}_{4}$ & 0,15 & 0,12 & 0,08 & 0,08 & $-0,03$ & $-0,06$ & 0,00 & 0,27 & 0,25 & 0,18 & 0,19 & 1,00 \\
\hline
\end{tabular}

Valores en rojo indican correlaciones significativas (Test de Kendall, $p<0,05$ ).

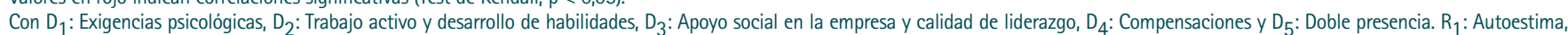
$\mathrm{R}_{2}$ : Impulsividad, $\mathrm{R}_{3}$ : Locus de control y $\mathrm{R}_{4}$ : Tensión. 
nado por Silva, Lefio, Marchetti y Benoit ${ }^{19}$, quienes en un estudio -cuyo objetivo era estimar la asociación entre factores de riesgo psicosocial presentes en el trabajo, la autopercepción de salud y siniestralidad laboral en conductores de vehículos de transporte de carga y pasajeros, urbanos e interurbanos en Santiago de Chile-, al comparar sus resultados con la referencia nacional obtenida por Alvarado, Marchetti, Villalón, Hirmas y Pastorino ${ }^{20}$, determinaron que la dimensión trabajo activo y desarrollo de habilidades resultó ser no significativa para la muestra en estudio. A su vez, los resultados coinciden con lo obtenido por Gómez, Hernández y Méndez ${ }^{21}$, quienes realizaron un estudio en una empresa chilena subcontratista del área de la minería, determinándose diferencias estadísticamente significativas dentro de los grupos en estudio en la dimensión trabajo activo y posibilidades de desarrollo en la variable cargo (jefatura/supervisor, profesionales, administrativos/ técnicos y operarios).

Rasgos de personalidad según condición de accidentabilidad En ambas condiciones de estudio se presenta una tendencia al locus de control externo (a mayor puntaje, el patrón externo se hace presente), resultado que concuerda con lo obtenido en un estudio sobre accidentes viales en el sur de Tamaulipas, México, en donde la existencia de un locus externo, determinó la responsabilización de factores distintos al conductor del incidente (clima, visibilidad, carretera, entre otros) ${ }^{22}$.

Los resultados demuestran que la frecuencia en los niveles de autoestima baja y alta es igual en ambos grupos de estudio, lo que concuerda con lo obtenido por Alonso $^{23}$, quien explica que la autoestima influye en el trabajo y el trabajo influye en la autoestima. El trabajador con un sentimiento positivo de sí mismo experimenta una mayor satisfacción y bienestar a través de su actividad laboral, al ocupar sus habilidades, desarrollar su potencial y realizarse en lo que hace, por lo que se va a auto-motivar, optimizando su desempeño; sin embargo, el trabajo puede no ser esa herramienta de desarrollo, sino muy por el contrario, una fuente de sentimientos negativos, frustración y malestar para el individuo.

Para la condición con accidente, los niveles de impulsividad y tensión son mayores que en el grupo sin accidente, aunque esta diferencia no fue estadísticamente significativa. Esto concuerda con lo obtenido por Hansen (1989); citado por Jaén ${ }^{24}$, quien identificó que dos tipos de personalidad eran los más propensos a tener accidentes: extravertidos socio-patológicos (centrados en sí mismos, confiados en exceso, agresivos, irresponsables, resentidos, intolerantes, impulsivos, antisociales y autoritarios), considerando como rasgo de personalidad la impulsividad; y los neuróticosansiosos (caracterizados por la tensión, excesivamente sensibles a las críticas, indecisos, incapaces de concentrarse, fatigados, alicaídos, emocionalmente inestables, con sentimientos de inadecuación), considerando como rasgo de personalidad la tensión. Un estudio realizado por Gálvez, Mingote y Moreno ${ }^{25}$, hace referencia a que las personas con trastornos de personalidad en el trabajo, con un estilo antisocial de personalidad, no les amedrentan las situaciones que asustan a la mayoría de las personas, el miedo es algo emocionante, y, por tanto, pueden cometer actos temerarios en el lugar de trabajo, lo que puede ser causa de accidente laboral. Son individuos que expresan abiertamente sus emociones, son ambiciosos, necesitan a su lado a personas que le den mucho a cambio de poco y, con frecuencia, no cumplen las normas, aunque pueden ser buenos trabajadores si la tarea les ofrece nuevos retos y estimulación novedosa de forma continua. Bajo un estrés persistente o especialmente agudo, pueden distorsionar la realidad para reducir la tensión y ansiedad, reaccionando con una respuesta exagerada e impulsiva, en la que muestran sus pensamientos, emociones y conducta de forma abierta, aunque esto sea ofensivo socialmente. Las personas con conductas violentas suelen tener una serie de características propias con trastornos de personalidad de tipo narcisista o antisocial, en la que el sujeto tiene una idea grandiosa de su propia importancia; le absorben fantasías ilimitadas de éxito y de poder; se considera especial y único: tiene una necesidad excesiva de ser admirado; puede fingir que entiende los sentimientos de los demás, y tiene actitudes y comportamientos arrogantes. Estas actitudes (con sus componentes cognitivos, emocionales y conductuales) en el lugar de trabajo pueden ser claramente desencadenantes o facilitadoras de conflictos interpersonales, situaciones de acoso, incremento del estrés psicosocial, mala distribución del trabajo, evasión de responsabilidades, entre otras; es decir, desencadenantes o facilitadoras de condiciones de trabajo con riesgo psicosocial.

Al comparar los puntajes de ambos grupos, y si bien no se manifestaron diferencias estadísticamente significantes, el grupo de trabajadores sin accidente presentó un mayor nivel de autoestima, tendencia al locus de control externo y mayor nivel de tensión comparado con el grupo de trabajadores con accidentes.

Para el caso de impulsividad, los resultados de este estudio coinciden con lo obtenido por Sannino, Gallardo, Ackerknecht y López-Mena ${ }^{26}$, quienes determinaron que no existe relación estadisticamente significativa entre presentar alta o baja impulsividad y haber sufrido un accidente o incidente dentro del último año. Respecto al locus de control, García-Layunta, Oliver, Tomás, Verdú y Zaragoza ${ }^{27}$ concluyeron que la realización de horas extras y el locus de control no presentaban relaciones estadísticamente significativas con la siniestralidad. En cuanto al nivel de autoestima, un estudio realizado por la Universidad de Zaragoza determinó que en las empresas ha predominado la creencia de que la siniestralidad laboral se debe principalmente a errores o equivocaciones humanas. Estas empresas se quejan de los trabajadores, de su escasa cualificación, de la falta de observación de las normas, de su desidia, entre otros; y cargan la culpa de los accidentes directamente sobre ellos. Esta postura está tan arraigada que incluso los mismos trabajadores que han sufrido un accidente llegan, en ocasiones, a desarrollar un sentimiento de culpa, una conducta de baja autoestima, al atribuir la responsabilidad del accidente a un error cometido por ellos mismos ${ }^{28}$.

\section{Relación entre variables socio-demográficas, dimensiones de riesgo psicosocial y rasgos de personalidad, según condición de accidentabilidad}

Los resultados de la relación entre Apoyo social y calidad de liderazgo refuta lo obtenido por Mena y Campos ${ }^{29}$, quienes concluyeron que el estrés percibido puede ser considerado como un buen indicador de estrés, asociándose con una serie de factores determinantes en el nivel de bienestar psicológico y físico del individuo. Si al nivel de estrés observado se le asocia la percepción de un apoyo social escaso, las posibilidades de desarrollo de patologías se ven incrementadas, puesto que el trabajador no encuentra las redes de apoyo social protector. Lo anterior concuerda con los resultados obtenidos por Güilgüiruca, Meza, Góngora y Moya $^{30}$, quienes, en un estudio en trabajadores de una empresa eléctrica en Chile, determinaron la presencia de una alta correlación entre apoyo social y exigencias 
psicológicas con el estrés percibido, indicando que a medida que se torna más crítica la percepción de apoyo de pares o del líder, más aumenta el estrés percibido por los trabajadores.

Las relaciones entre variables sociodemográficas, dimensiones de riesgo psicosocial y rasgos de personalidad son argumentadas por Acevedo, Sánchez, Farías y Fernández ${ }^{31}$ y Güilgüiruca, Meza, Góngora y $\mathrm{Moya}^{30}$, quienes describen la relación entre la antigüedad laboral y doble presencia, concluyendo que trabajadores con menor antigüedad perciben una mayor interferencia del trabajo con su vida privada. Del mismo modo, los últimos autores confirman una estrecha relación entre estrés percibido y doble presencia.

Zapata, Jiménez y Ramírez ${ }^{32}$ analizaron las variables psicológicas que explicaban la ocurrencia de accidentes en trabajadores de cosecha forestal en la región de la Araucanía, Chile. En dicha investigación se obtuvo como resultado que los sujetos en estudio que se accidentaban presentaban ciertos factores gatillantes en su faena, tales como: la presión ejercida por los compañeros de trabajo, las expectativas de sí mismo y su familia, las que se relacionan con la motivación a maximizar la productividad, asociándose a una menor atención y concentración en la tarea. La mejor opción para generar resultados es en la medida que aumentan las expectativas del trabajador, interés por superar a otros y/o a sí mismo, y reconocimiento social; una valoración intuitiva negativa del peligro, considerando el grado de control que pudiera ejercer sobre él y el nivel de conocimiento o desconocimiento del mismo. Este factor se evidencia principalmente en sujetos que no han vivenciado ni experimentado accidentes, a partir de lo cual son propensos a realizar acciones por el solo hecho de complacer los requerimientos de aquellos considerados superiores. Estos autores aseveran que los sujetos muestran una imagen de sí mismos variable y relacionada específicamente con dos aspectos fundamentales: el logro, en términos de la obtención de bienes materiales y de un status preponderante en relación al grupo; es así como, en determinadas circunstancias, los sujetos exhiben una mayor seguridad y mayor autoestima (degradación baja) y, en otras, emerge un sentimiento de inferioridad respecto de los demás, o de aquellos considerados superiores, jerárquicamente o en relación a sus destrezas; una elevada necesidad de proteger, dada por la posición social y la presión que ésta ejerce sobre los hombres con respecto a su rol, influyendo en las conductas tendientes a buscar formas de contener, tanto afectiva, como económicamente a la familia; la necesidad de autonomía, relacionada a su vez con la autoestima de los sujetos, ya que la posibilidad de realizar acciones de manera independiente, sin contar con respaldo, está relacionada con la imagen que los sujetos tienen de sí mismos y la valoración que estos realicen de sus habilidades y destrezas a la tarea, así como de las experiencias previas en la ejecución, ya sea de sí mismos como de otros considerados referentes.

En conclusión, se presentaron diferencias significativas entre los grupos con y sin accidentes, sólo en una dimensión de riesgo psicosocial, siendo esta trabajo activo y desarrollo de habilidades. Para ambos grupos en estudio, según condición de accidentabilidad, se obtuvo igual número de conductores que poseían autoestima baja y alta. Para la condición con accidente, el mayor número de casos fue clasificado con rasgo de personalidad impulsiva, a diferencia de la condición sin accidentes, quienes poseían un mayor número de casos con locus de control externo. Para el rasgo de personalidad tensión, en ambos grupos prevaleció la ausencia de estrés. Para el grupo sin accidente, se presentó un alto porcentaje de casos con estrés agudo, en comparación con los otros niveles de estrés, a diferencia del grupo con accidente, en los que predomina el nivel de estrés funcional. Para el grupo con accidentes, trabajo activo y desarrollo de habilidades fue la dimensión que presentó un mayor número de correlaciones significativas, tanto con otras dimensiones de riesgo psicosocial, así como también con estrés percibido; en cambio, en el grupo sin accidentes, el mayor número de correlaciones significativas se presentó entre doble presencia y los rasgos de personalidad impulsividad, locus de control y tensión. 
1. Berruezo D. Causalidad de los accidentes de trabajo [en línea]. Barcelona: FFH; 2002 [citado 22 sep 2014]. Disponible en: http://www.factorhuma.org/index. php?option=com_content\&view=article \&id=6696\%3ACausalidad $+d e+l o s+a c$ cidentes+de+trabajo\&catid=4\%3Anoticias\&ltemid=11\&lang=es

2. Díaz C, Galetovic A, Sanhueza R. La regulación del transporte de carga en Santiago: Características, evaluación y propuestas. Cuad Econ. 2003; 40(119):5-46.

3. Puente-Rodríguez E, Pillon S. Estrategias de prevención y vigilancia sobre el uso de sustancias ilícitas y su impacto en los costos de accidentes en conductores de carga pesada en México. Rev Latino-Am Enfermagem. 2011; 19:831-838.

4. Zaldivar M. Estadisticas de accidentabilidad 2013 [en línea]. Santiago de Chile: Gobierno de Chile; 2014 [citado 13 sep 2014]. Disponible en: http:// 163.247.55.110/PortaIWEB/SST/estadisticas/2014_04_28_Estad\%C3\% ADsticas\%20de\%20Accidentabilidad\%202013.pdf

5. Reyes R, Prado L, Aguilera A, Soltero, R. Descripción de los conocimientos sobre factores humanos que causan accidentes en una industria arnesera mexicana. e-Gnosis. 2011; 9:1-17.

6. Feldmam L, Blanco G. Una aproximación al estudio de los factores psicosociales laborales en Venezuela. Salud trab. 2012; 20(1):75-92.

7. Chile. Ministerio de Salud. Encuesta nacional de empleo, trabajo, salud y calidad de vida de los trabajadores y trabajadoras (ENETS) [en línea]. Santiago de Chile: MINSAL; 2011 [citado sep 2017]. Disponible en: http://www.isl.gob.cl/ wp-content/uploads/2011/09/Informe-Final-ENETS-Interinstitucional.pdf

8. Chile. Ministerio de Salud. Protocolo de Vigilancia de Riesgos Psicosociales en el trabajo [en línea]. Santiago de Chile: MINSAL; 2013 [citado sep 2017]. Disponible en: http://web.minsal.cl/portal/url/item/e039772356757886e04001 0165014a72.pdf

9. Martínez $\mathrm{S}$, Hernández $\mathrm{A}$. Necesidades de estudios y legislación sobre factores psicosociales en el trabajo. Rev Cubana Salud. 2005; 31(4):338-344.

10. Sánchez A. Rasgos de personalidad relacionados con la ocurrencia de accidentes de trabajo de riesgo biológico en el personal de enfermeria del Hospital Universitario de Santander (HUS) 2008-2009 [tesis en linea]. Bucaramanga: Universidad Industrial de Santander; 2011 [citado sep 2017]. Disponible en: http://tangara.uis.edu.co/biblioweb/tesis/2011/140869.pdf

11. Soler M. La evaluación de los factores de riesgo psicosocial del trabajo en el sector hortofruticola: el cuestionario de FAPSIHOS [en línea]. Murcia: Universidad de Murcia; 2008 [citado oct 2017]. Disponible en: http://www.tdx. cat/bitstream/handle/10803/11033/SolerSanchez.pdf;jsessionid=E94A1FDA7C1 45B8B51EF870BCB8C786D.tdx2? sequence $=1$

12. Rojas-Barahona C, Zegers B, Förster C. La escala de autoestima de Rosenberg: Validación para Chile en una muestra de jóvenes adultos, adultos y adultos mayores. Rev méd Chile. 2009; 137:791-800.

13. Salvo L, Castro A. Confiabilidad y validez de la escala de impulsividad de Barratt (BIS-11) en adolescentes. Rev chil neuro-psiquiatr. 2013; 51(4):245-254.

14. Alvarado R. El Rol del Locus de Control como factor moderador en trastornos ansiosos y depresivos en los adultos mayores. PSYKHE. 2003; 12(1):57-64.

15. Meyer A, Ramírez L, Pérez C. Percepción de estrés en estudiantes chilenos de medicina y enfermería. Rev educ cienc salud. 2013; 10(2):79-85.

16. Montgomery D. Diseño y análisis de experimentos. México DF: Grupo Iberoamericano S.A.; 1991.
17. Steel $R$, Torrie J. Bioestadística: Principios y Procedimientos. $2^{\mathrm{a}}$ ed. México DF: McGraw-Hill; 1997.

18. Corzo J. Estadistica no paramétrica: Métodos basados en rangos. Bogotá: Universidad Nacional de Colombia; 2005.

19. Silva H, Lefio A, Marchetti N, Benoit P. Riesgos Psicosociales en Conductores de Transporte de Carga y Pasajeros Urbanos e Interurbanos, y su Asociación con la Autopercepción de Salud y Siniestralidad Laboral. Cienc Trab. 2014; 16(50):67-74.

20. Alvarado R, Marchetti N, Villalón M, Hirmas M, Pastorino M. Adaptación y análisis psicométrico de un cuestionario para evaluar riesgos psicosociales en el trabajo en Chile: Versión media del CoPso0. Rev chil salud pública. 2009; 13(1):7-16.

21. Gómez P, Hernández J, Méndez, M. Factores de Riesgo Psicosocial y Satisfacción Laboral en una Empresa Chilena del Área de la Minería. Cienc Trab. 2014; 16(49):9-16.

22. Carro E, Spinoso P, Esteves Z, Castellanos G. Locus de control y accidentes viales publicados en la prensa escrita del sur de Tamaulipas [en linea] Tamaulipas: Universidad Autónoma de Tamaulipas; 2012 [citado sep 2017]. Disponible en: http://www.eumed.net/libros-gratis/2013a/1336/control-accidentes.html

23. Alonso A. Factor individual Autoestima. Curso de enfermería de empresa. Salamanca: [e.d]; 2003 [citado sep 2017]. Disponible en: http://www.enfermeriasalamanca.com/TRABAJOS_SALUD_LABORAL/SEGURIDAD/AUTOESTIMA.PDF

24. Jaén M. Predicción del rendimiento laboral a partir de indicadores de motivación, personalidad y percepción de factores psicosociales [en línea]. Madrid: Universidad Complutense de Madrid; 2010 [citado oct 2017]. Disponible en: http://eprints.ucm.es/10843/1/T31913.pdf

25. Gálvez M, Mingote J, Moreno B. El paciente que padece un trastorno de personalidad en el trabajo. Med segur trab. 2010; 56(220):226-247.

26. Sannino D, Gallardo M, Ackerknecht C, López-Mena L. Evaluación conductual de la impulsividad aplicada al control de la accidentalidad. Cienc Trab. 2012; 43:105-111.

27. Garcia-Layunta, M, Oliver A, Tomás J, Verdú F, Zaragoza, G. Factores psicosociales influyentes en la ocurrencia de accidentes laborales. Arch prev riesgos labor 2002; 5(1): 4-10.

28. Puyal. La conducta humana frente a los riesgos laborales. Determinantes individuales y grupales. Acciones invest soc [en línea]. 2001 [citado 23 dic 2014]. Disponible en: https://papiro.unizar.es/ojs/index.php/ais/article/viewFile/199/193

29. Mena L, Campos J. Evaluación de Factores presentes en el estrés laboral. Rev Psicol (Santiago Chile). 2002; 11(1):149-163.

30. Güigüiruca M, Meza K, Góngora R, Moya $C$. Factores de riesgo psicosocial y estrés percibido en trabajadores de una empresa eléctrica en Chile.; Rev med segur trab. 2015; 61(238):57-67.

31. Acevedo G, Sánchez J, Farias M, Fernández A. Riesgos psicosociales en el equipo de salud pública de hospitales públicos de la provincia de Córdova, Argentina. Cienc Trab. 2013; 15(48):140-147.

32. Zapata $P$, Jiménez $C$, Ramírez C. Análisis de las variables psicológicas que explican la ocurrencia de accidentes entre trabajadores de cosecha forestal en la Región de la Araucanía [tesis en línea]. Santiago: Universidad de Chile; 2008 [citado oct 2017]. Disponible en: http://www.tesis.uchile.cl/tesis/uchile/2008/ carrasco_m/sources/carrasco_m.pdf 